Europhys. Lett., 19 (3), pp. 171-176 (1992)

\title{
2D Dynamics of a Drop Falling in a Miscible Fluid.
}

\author{
A. Garcimartín, H. L. Mancini (*) and C. PÉrez-García (**) \\ Departamento de Física y Matemática Aplicada, Facultad de Ciencias \\ Universidad de Navarra - 31080 Pamplona, Spain
}

(received 13 January 1992; accepted in final form 27 April 1992)

PACS. 47.10 - General theory.

PACS. 47.20 - Hydrodynamic stability and instability.

PACS. 68.10 - Fluid surfaces and interfaces with fluids.

\begin{abstract}
The dynamics of the fragmentation of a liquid drop falling in a miscible fluid is studied in a Hele-Shaw geometry. We show that this configuration allows quantitative measurements of the characteristic sedimentation velocity and internal velocity of the drop. The break-up process observed in $3 \mathrm{D}$ is also found in this quasi-2D configuration. The relation between the internal circulation pattern and this process is also discussed.
\end{abstract}

Fluid instabilities occur in many natural phenomena (flows in porous media, dynamics of earth mantle, atmospheric currents) as well as industrial processes (stirring tanks, mixing processes). In the last few years considerable attention has been devoted to basic hydrodynamical instabilities (convective, centrifugal, gravitational) that could offer insight on the basic instability mechanisms under quite well-regulated conditions.

Instabilities can be found even at small Reynolds number and for open flows. One of the simplest instabilities of this kind is the fragmentation of a drop falling in a miscible fluid. This instability was first described by von Helmholtz and inspired Lord Kelvin his works on vorticity. A detailed phenomenological description can be found in ref. [1]. These authors reported the fragmentation process in a wide variety of miscible liquids and also discussed the case of immiscible liquids. The process could be summarized as follows. A drop is deposited on the free surface of a lighter liquid. After a short period, it begins to fall, leaving a tail at the rear. After some time the drop breaks up forming a torus that expands radially. This torus in turn is unstable and undergoes a fragmentation in several drops, and these daughter drops follow the same process again. The process is the same if the densities of the fluids are reversed, the drop then moving upward.

Recent research $[2,3]$ has thrown light on the phenomenon. The break-up process is explained in terms of competition between diffusion and viscous transfer of momentum along the radius. If the second one is faster than the first, the drop breaks up. But diffusion efficiency grows as the area increases and the transfer rate of velocity is inversely proportional to the radius, so the fragmentations eventually stop and diffusion becomes dominant. Moreover Baumann et al.

(*) Also at CITEFA/CONICET (1603 V. Martelli) and Universidad de San Luis, Chacabuco y Pedernera, 5700 San Luis, Argentina.

(**) Also at Universitat Autónoma de Barcelona, Departament de Física, 08193 Bellaterra, Catalonia, Spain. 
showed in a recent paper [4] that the break-up process can also occur in immiscible fluids when viscous effects are considerably bigger than those resulting from surface tension, contrary to the statements in ref. [1]. The question has been raised about the existence of dynamical interfacial forces $[5,6]$, which would appear even between miscible fluids. These forces would arise as a result of gradients of physical quantities at the interface.

However, a detailed theoretical description of the fragmentation is still lacking. The formation of the drop on the free surface of another liquid is an example of Rayleigh-Taylor instability, and the deformation and fragmentation of the drop can be regarded as a special case of Kelvin-Helmholtz instability. Mathematical difficulties arise when trying to carry out the stability analysis for a nonspherical geometry including viscous effects. Kojima et al. [7] have calculated numerically the shape of the drop during the early stages of its deformation. Pozrikidis [8] took a further step, adding nonlinear terms to that analysis. Nevertheless these works do not explain the whole fragmentation process.

To investigate the physical origin of this instability, we have carried out some experiments in a Hele-Shaw cell wide enough to accommodate the drop but at the same time narrow enough to prevent the formation of a torus. (The width of the cell is of the same order than the radius $r$ of the drops.) Thus, while imposing mild restrictions on the dynamics (velocity, distance and time scales), it is more easily shown what is going on inside the drop. The set-up provides in this way a kind of vertical section of the process. Of course this imposes a restriction on $r$, but we arranged several cells with different widths to overcome the problem. The cell is made from plexiglass and the liquids employed are glycerin-water mixtures in different proportions.

We recorded the process by means of a CCD camera to allow better control of timing. Thus we could replay the whole process in slow motion, with a resolution better than one-tenth of second for the faster drops. Volumes were controlled by a microsyringe with an accuracy better than $0.2 \mu \mathrm{l}$ : the uncertainties in the value of the radius-the relevant parameter-are therefore pretty small. In some cases, small quantities of food colourant were used to improve contrast without significant influence on the process. In other cases, we seeded drops with latex particles to allow flow visualization. Latex density can be customized, then fluid properties are not severely altered. With an image processing system they are easily detected and tracked, and therefore quantitative data can be recorded.

We notice that the initial shape of the drop is not well defined and sometimes is completely irregular, due to the geometrical constraints. However, when the drop enters into the lighter fluid a quasi-spherical form is quickly achieved (see fig. 1). The drops begins to fall and after a short transient a sedimentation velocity is reached. In fig. 2 we plot the measured velocity of typical drops in our experiments. The points correspond to the velocity averaged over 10 drops of the same density and size and the solid line gives the linear fitting until the drop starts its horizontal deformation. The origin of distances is an arbitrary point near the surface of the liquid. For comparison we also plotted the sedimentation velocity corresponding to a solid sphere that obeys the Stokes law (drag force $F=6 \pi \mu r v$ ). Considering that in the experiment the drop is not solid, mass is not conserved and its density and radius are changing, it is remarkable that the values on these two lines differ only about $15 \%$. The important conclusion is that an approximately constant sedimentation velocity exists, which lies close to that calculated by the Stokes law even without including the correction term $(2 / 3+\lambda) /(1+\lambda)$, $\lambda$ being the ratio between the $\operatorname{drop}\left(\mu_{2}\right)$ and the liquid $\left(\mu_{1}\right)$ viscosities $\left(i=\mu_{2} / \mu_{1}\right)[9]$.

While the drop is falling a pattern of internal velocity is formed and can be determined from the latex particles trajectories. In the first stages of the evolution, the streamlines closely resemble the Hadamard-Rybczynski flow [10] for undeformed spherical drops. A stagnation point should appear inside the drop located at $r / \sqrt{ } 2$. We have measured it to be placed at $(0.7 \pm 0.1) r$. Therefore we conclude that this scheme of flow is valid as a first approximation. Once the drop begins to deform a suction zone at the rear of the drop due to a protrudence of the surrounding liquid inside the drop is 


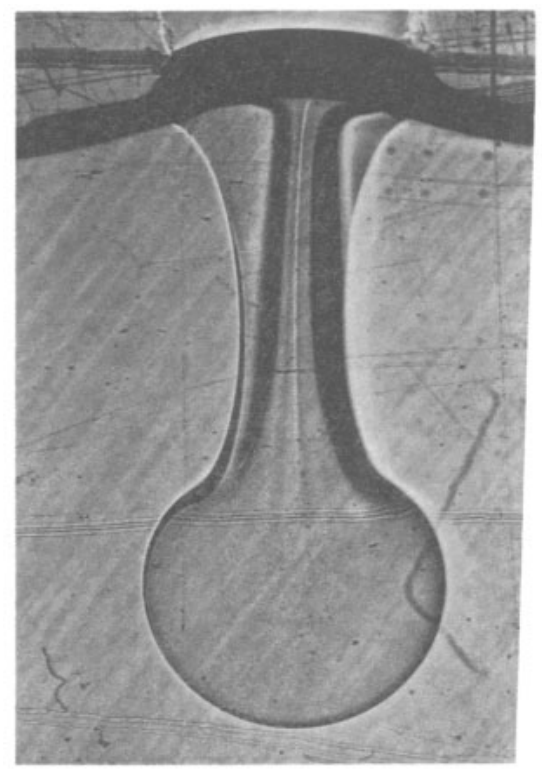

Fig. 1. - A drop in the first stages of evolution. The dark stripe on the top is the free surface of the liquid. The diameter of the drop is about $1 \mathrm{~mm}$.

visible. This coincides with theoretical predictions on flows in deformable drops (compare our fig. 3 with fig. 2 and 3 in ref. [8]). However, the main difference between the theoretical schemes [7,8] and experiments in miscible fluids is that the drop mass (including the tail) is not conserved.

After this deformation, the beginning of an interesting process can be seen near the tail attachment. Part of the liquid surrounding the drop travels along the streamlines nearly up to the tail, and another part enters inside the drop as is clearly visible in fig. 4a). (This incoming liquid appears as clear zones in the photographs.) The liquids do not mix themselves in a straightforward way, but rather the lighter liquid enters the drop following spiral paths (see fig. $4 b$ )). Thus the mixing takes place inside the drops. Meanwhile the drop begins to split into two parts and the centre of mass is displaced outwards. A complex motion near the bottom of the drops tends to change the curvature of the interface between the drop and the surrounding fluid: it changes from

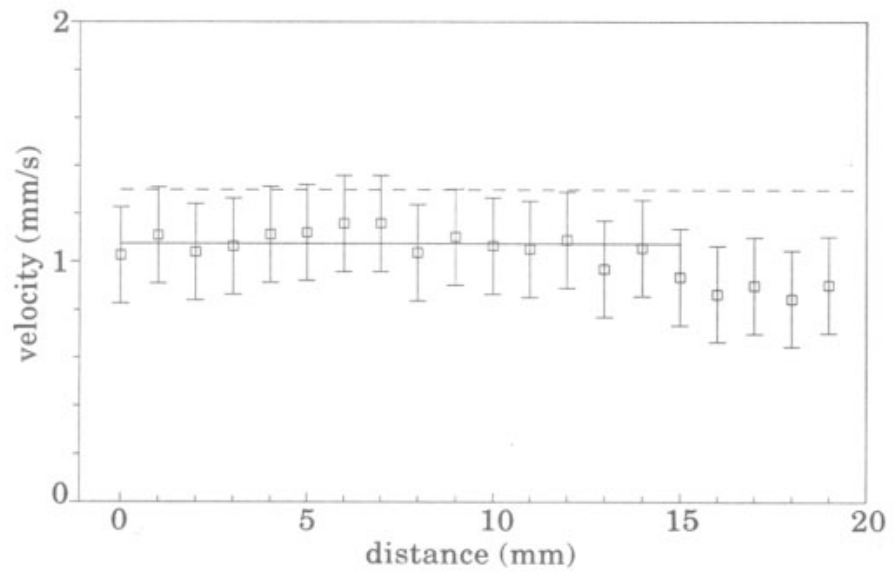

Fig. 2. - Sedimentation velocity of the drop. The dashed line represents Stokes sedimentation for a solid sphere. The solid line is the linear fit for the drop velocity before its diameter begins to increase noticeably. 


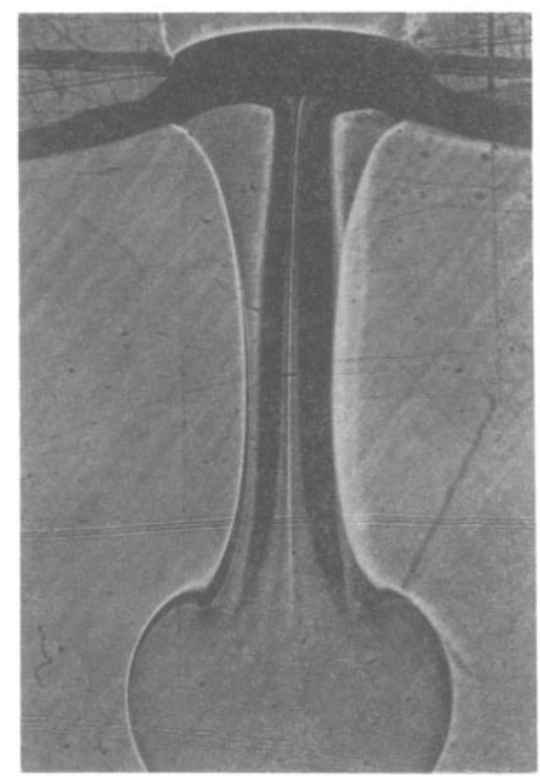

Fig. 3. - The rear part of the drop shows suction zones near the tail. From this point on mixing with external fluid begins. Compare this photograph with fig. 3 from ref. [8].

convex (fig. 4a)) to concave (fig. 4b)). This leads to a break-up process of the drop into two daugther drops of approximately the same volume (see fig. $4 b$ )) that still remain in contact through a thin filament. (These features are similar to the break-up process of a torus in $3 \mathrm{D}$, although more than two fragments can be obtained in this case [3].)

It is worth noting that this evolution is quite typical, i.e. it is similar for different values of the parameters that characterize the drop. It is also valid for drops of lighter fluid that go up instead of falling in the surrounding liquid. Typical velocities inside the drop are related to the sedimentation velocity $v_{\infty}$ by the relationship $v_{\mathrm{i}}=v_{\infty}[-1 / 3(1 /(2+3 \lambda))]$. This velocity $v_{\mathrm{i}}$ can be considered as a reference value for the intermal velocity field. (A new stable sedimentation velocity is quickly reached after fragmentation; the transient time is much smaller than the elapsed time till break-up $\left(\tau_{\text {bu }}\right)$ in our experiments.) The fragmentation takes place when internal velocity and mixing process are developed inside the drop; this therefore suggested that internal velocity and break-up were

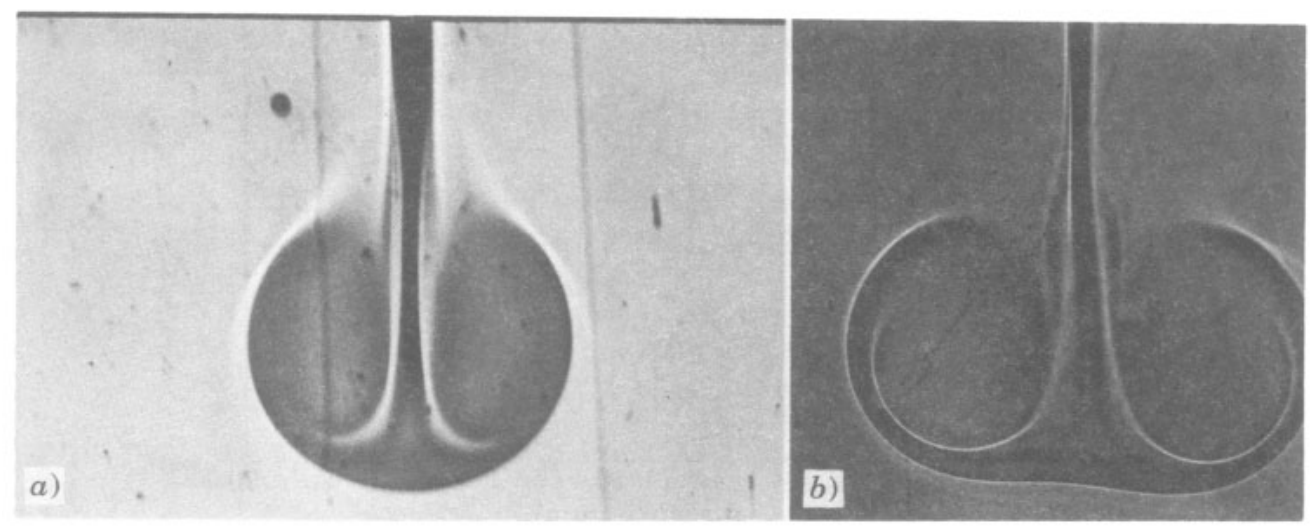

Fig. 4. - a) Lighter stripes of fluid can be seen entering the drop. Mixing takes place inside the drop. b) Spiral paths of the fluid entering inside the drop. The drop is deformed into two parts of approximately the same volume. 
somehow related. The pattern of the incoming fluid also suggests that the mechanism of the break-up is linked with the formation of the filaments that connect the daughter drops. Therefore, the time for the break-up process should be related with the time taken by the external fluid to configure these streamlines.

To substantiate the above argument we compared break-up times with the characteristic time of the internal velocity pattern. Arecchi et al. [3] had already suggested that $\tau_{\mathrm{bu}}$ should scale as $r / v_{\infty}$. In fact $\tau=r / v_{\infty}$ could be considered as the characteristic time of viscous velocity transfer across the radius of the drop.

But it should be noticed that, although the viscosity ratio does not affect the sedimentation velocity essentially, it introduces a quantitative difference between external and internal velocities (the latter depends on the viscosity ratio). Then $\tau$ must be replaced by an internal typical time $\tau_{\mathrm{i}}=$ $=r / v_{\mathrm{i}}$. It is easy to see that the latter can be interpreted as the typical time necessary for a fluid particle to follow a complete streamline inside the drop. $\tau_{\mathrm{i}}$ is also the typical time to establish the streamline of the incoming fluid. If this were linked with the mechanism of the break-up process, a simple relationship between $\tau_{\mathrm{bu}}$ and $\tau_{\mathrm{j}}$ should exist. We tested this conjecture for very different times of break-up, ranging from less than one second to more than sixty seconds. The main result is shown in fig. 5 , where an almost linear relationship between the measured $\tau_{\mathrm{bu}}$ and $\tau_{\mathrm{i}}=r / v_{\mathrm{i}}$ is obtained. The ratio between these variables is fairly close to one.

The main source of error and the reason for some of the gaps in the graph is the following. When both viscosities are of the same order, even strong mixing will leave break-up characteristic times unaffected. However a great uncertainty arises when viscosity differences between the drop and the surrounding liquid are very big, because the mixing process can change initial values significantly. This happens because viscosity of glycerin-water mixtures increases exponentially with glycerin proportion and so a small income of mass can change the difference of viscosities drastically. For instance, if the volume ratio glycerin/water is changed only $5 \%$, viscosity could change in some cases 50\% [11]. Therefore in these cases one should search for those combinations with small break-up times to prevent mixing as much as possible. This conditioned the mixtures that could be studied quantitatively. For some liquid proportions uncertainties were so big that although measurements yielded consistent results they were discarded.

The daughter drops formed after fragmentation separate quickly apart (see fig. 6). For

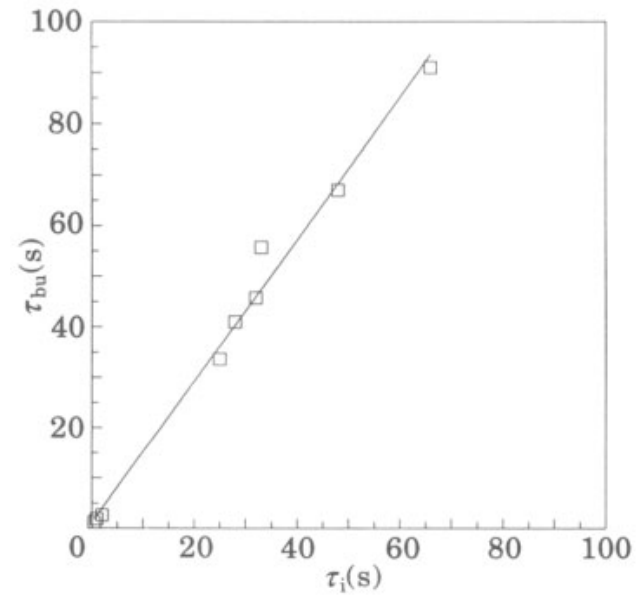

Fig. 5.

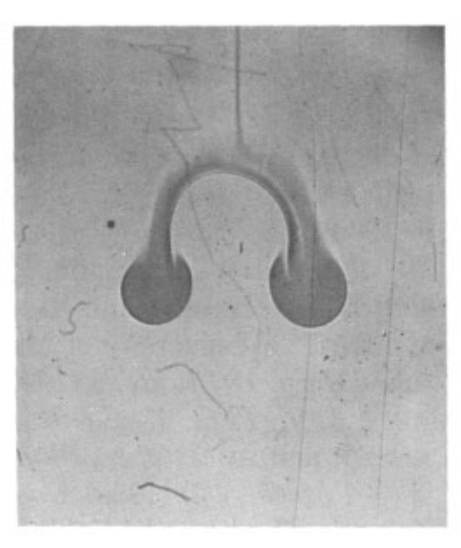

Fig. 6.

Fig. 5. - Experimental break-up times $\left(\tau_{\text {bu }}\right) v s$. internal viscous time $\left(\tau_{\mathrm{i}}=r / v_{\mathrm{i}}\right)$. An almost linear relationship is found between them. Best fit: $\tau_{\mathrm{bu}}=1.14 \cdot \tau_{\mathrm{i}}$.

Fig. 6. - Two secondary drops separate quickly a short time after break-up. 
instance, for a typical drop of $r=1 \mathrm{~mm}$ falling at about $v=1 \mathrm{~mm} / \mathrm{s}$ the two fragments separate about 4 or $5 \mathrm{~mm}$ in a few seconds. The horizontal velocity in this process is of the same order as the sedimentation velocity. During this stage these drops show a kind of rigid-body-like rotation. The hydrodynamical mechanism for this effect is not clear. Although a Magnus force mechanism could be proposed, simple calculations show that this force is not enough to account for this separation process. This lateral displacement stops when a circulation pattern similar to that in the initial drop is formed in the daughter drops. We have empirically fitted the paths followed by the daughter drops at this stage and we have found that they are nearly parabolae. When the internal velocity pattern is established inside the drops, they go on falling vertically. In fact these daughter drops are constituted mainly by the unmixed fluid of the primary drop. A noticeable amount of strongly mixed matter is released to the surrounding fluid. After some time the flow inside these drops changes from a rigid-body-like rotation to a flow similar to the Hadamard-Rybzinsky pattern and the incoming fluid also destabilizes these secondary drops. The process is repeated until the fragments formed are so small that they fail to develop an internal circulation and they do not break any more. In our experiments we never observed more than four successive break-ups.

We thus conclude that the formation and evolution of the velocity field inside the drop is a key point for the understanding of the break-up process. The instability is seemingly related with the mixing process of the lighter fluid coming inside the drop. The fact that the elapsed time until the break-up is almost the same as the typical time of internal circulation supports this interpretation; and a quantitative evidence has been presented. We also highlight that the results presented here agree qualitatively with similar studies in $3 \mathrm{D}[2,3]$, but give a more precise idea of the time scales that rule the processes, and reinforce the conjecture that the inertia forces are important in the fragmentation mechanism [4].

We have benefitted greatly from the technical support and comments of J. BURGUETE and P. ELIZALDE. We also thank J. E. WESFREID (Paris) for his stimulating suggestions. One of us (HLM) acknowledges DGICYT (Spanish Government) for a sabbatical year. This work has been partially supported by DGICYT OP90-0098 (Spanish Government) and by OF725/91 (Gobierno de Navarra).

\section{REFERENCES}

[1] Thomson J. J. and Newall H. F., Proc. R. Soc. London, 39 (1885) 447.

[2] Arecchi F. T., Buah-Bassuah P. K., Francini F., Pérez-García C. and Quercioli F., Europhys. Lett., 9 (1989) 333.

[3] Arecchi F. T., Buah-Bassuah P. K. and PÉrez-García C., Europhys. Lett., 15 (1991) 429.

[4] Baumann N., Joseph D. D., Mohr P. and Renardy Y., Vortex Rings of One Fluid in Another in Free Fall, preprint IMA 783, University of Minnesota, Feb. 1991.

[5] Joseph D. D., Eur. J. Mech. B/Fluids, 9 (1990) 565.

[6] May S. E. and Maher J. V., Phys. Rev. Lett., 67 (1991) 2013.

[7] Kojima M., Hinch E. J. and Acrivos A., Phys. Fluids, 27 (1984) 19.

[8] Pozrikidis C., J. Fluid Mech., 210 (1990) 1.

[9] Happel J. and BREnNer H., Low Reynolds Number Hydrodynamics (Noordhoff International Publishing, Leyden) 1973.

[10] Haddamard J., C. R. Acad. Sci. Paris, 152 (1911) 1735; Rybczynski W., Bull. Acad. Sci. Cracovie, 1 (1911) 40.

[11] Hellwege K. H. (Editor), Landolt-Börnstein Numerical Data and Functional Relationships in Science and Technology, Vol. 5, Band 2 (Springer-Verlag, Berlin) 1979. 